Research Article

\title{
Prediction of Mine Dust Concentration Based on Grey Markov Model
}

\author{
Zhou Xu, ${ }^{1}$ Guo Liwen, ${ }^{1}$ Zhang Jiuling ${ }^{10},{ }^{1}$ Qin Sijia, ${ }^{2}$ and $\mathrm{Zhu} \mathrm{Yi}^{2}$ \\ ${ }^{1}$ College of Mining Engineering North China University of Science and Technology, Tangshan 063210, China \\ ${ }^{2}$ Yisheng Innovation Education Base North China University of Science and Technology, Tangshan 063210, China
}

Correspondence should be addressed to Zhang Jiuling; ninety2000@163.com

Received 20 September 2021; Revised 28 November 2021; Accepted 2 December 2021; Published 23 December 2021

Academic Editor: Tao Meng

Copyright $\odot 2021$ Zhou Xu et al. This is an open access article distributed under the Creative Commons Attribution License, which permits unrestricted use, distribution, and reproduction in any medium, provided the original work is properly cited.

\begin{abstract}
Accurate quantitative analysis and prediction of dust concentration in mines play a vital role in avoiding pneumoconiosis to a certain extent, improving industrial production efficiency, and protecting the ecological environment. The research has farreaching significance for the prediction of dust concentration in mines in the future. Aiming at the shortcomings of the grey GM $(1,1)$ model in forecasting the data sequence with large random fluctuation, a grey Markov chain forecasting model is established. Firstly, considering the timeliness of monitoring data, the new dust concentration data is supplemented by using the method of cubic spline interpolation in the original data sequence. Therefore, the GM $(1,1)$ model is established by the method of metabolism. Then, the GM $(1,1)$ model is optimized by the theory of the Markov chain model. According to the relative error range generated during the prediction, the state interval is divided. Subsequently, the corresponding state probability transition matrix is constructed to obtain the grey Markov prediction model. The model was applied to the prediction of mine dust concentration and compared with the prediction results of the BP neural network model, grey prediction model, and ARIMA (1,2, 1) model. The results showed that the prediction accuracy of the grey Markov model was significantly improved compared with other traditional prediction models. Therefore, the rationality and accuracy of this model in the prediction of mine dust concentration were verified.
\end{abstract}

\section{Introduction}

The continuous development of industrial technology and high technology has made the global economy develop rapidly and brought great convenience to our lives. However, in industrial production, with the improvement of coal mine tunneling and mining mechanization level, the dust production of mine working face is increasing [1]. In mine accidents, the occurrence of most accidents is related to the dust concentration in the mine. Mine dust will not only damage the mechanical life and endanger the safety and health of miners but also cause explosions when the dust reaches a certain concentration and even cause more serious underground disaster accidents [2]. Therefore, mine dust prevention work is becoming more and more important; the correct prediction of mine dust concentration is of great significance to the safe production under the mine.
Domestic and foreign scholars have conducted a lot of research on the prediction of mine dust concentration, and neural network, grey prediction model, and time series prediction model are widely used prediction methods. Wang [3] analyzed the influencing factors of dust concentration in the fully mechanized working face of coal roadway and used LMBP neural network to predict the dust concentration. Zhao and Ma [4] and others used the BP neural network optimized by particle swarm optimization algorithm to predict the dust concentration. $\mathrm{Li}$ [5] quantitatively analyzed the dust in the construction stage and used BP neural network to predict the concentration of PM10 so as to provide targeted protection measures for practitioners and provide effective protection for the health of construction personnel. Wang [6] et al. established a grey-generalized regression neural network combination model to study the accuracy of the model in predicting pneumoconiosis. 
However, the neural network model needs a large number of original sample data for long-term training and optimization, and the results are satisfactory, and the prediction ability for fluctuation data is generally weak.

Li [7] and Zhao [8] established a grey prediction model to predict and comprehensively evaluate the dust concentration and the operation status of petrochemical equipment. Wang [9] used the grey prediction model GM $(1,1)$ to predict and optimize PID optimization, which improved the accuracy of prediction data and the accuracy of shape control. Many experts and scholars have also continuously explored and improved the grey theory $[10,11]$. However, the grey $\operatorname{GM}(1,1)$ model is based on the exponential curve, which has poor fitting accuracy for large random fluctuation prediction objects. Through accumulation of the original data, its volatility is weakened. There is a certain deviation between the prediction results and the actual value.

Wang et al. [12] used the research method of time series to construct the prediction model of mine dust. Although the traditional time series model has a good fitting effect on the data samples with time variables as independent variables, it can only achieve short-term prediction, and the error increases rapidly when predicting.

Although the above model shows high fitting and accuracy in the field of traditional fitting prediction, due to the forgetting mechanism in the model, there will be some errors in the prediction of this kind of data with obvious time series characteristics. Liu et al. [13] constructed a grey prediction model based on the direct reporting data of the new coronavirus epidemic in Hubei Province from March 1 to March 21, 2020, and used the Markov model to correct the prediction results to predict the daily discharge number in Hubei Province from March 22 to March 24. It is concluded that the prediction accuracy of the grey Markov model is higher than that of the single grey prediction model. Shi $[14,15]$ established a grey Markov model to explore its application in the field of occupational disease prediction. Lu et al. [16], Wang and Peng [17], Weng et al. [18], and other people apply the grey Markov model to the prediction of subway passenger flow, production safety accidents, and surface subsidence. It is concluded that the grey Markov model is better than the prediction accuracy of the traditional grey GM $(1,1)$ model and more in line with the actual situation.

At the same time, the grey Markov prediction model can analyze the data with a small amount of data and high prediction accuracy and can handle the data with large random fluctuation $[19,20]$, but it has not been applied in the field of mine prediction.

In order to solve these problems, based on the dust concentration of a mine, this paper constructs the grey Markov model and the neural network model and compares them with the ARIMA model and the grey prediction model. The results show that the grey Markov model is superior in the prediction of dust concentration.

The rest of this article is organized as follows. Section 2 introduces the basic theory, including the neural network model, grey model, and Markov model. Section 3 uses ARIMA, neural network model, grey prediction model, and grey Markov model to predict and compare mine dust concentration. Section 4 solves the measurement performance evaluation index and tests the residuals of the four types of models. Finally, Section 5, draws conclusions, finds the most suitable evaluation model, and makes suggestions for future work.

\section{Theoretical Basis of the Model}

2.1. Theoretical Basis of the Neural Network. The learning and training process of the neural network method includes two stages: forward propagation and backward propagation. In the forward propagation process, the sample data are transmitted from the input layer to the output layer through the transfer function of the hidden layer [4]. If the output layer does not get the required output, it enters the backward propagation process and returns the error signal along the original forward propagation path. The mean square error and gradient descent method are used to modify the network connection weight and adjust the mean square error between the actual output of the network and the guided learning signal $[21,22]$. This process has to be repeated until the specified error requirements or the maximum number of training are reached [23]. Figure 1 shows the concept map of neural networks.

2.2. Grey $\operatorname{GM}(1,1)$ Prediction Model. Grey system theory is a kind of control theory proposed by Professor Deng [24]. It takes the grey system as the research object and makes scientific quantitative predictions for the prediction and control of the development of various grey systems. It is widely used in agriculture, industry, meteorology, and other fields $[25,26]$. The concrete steps of the grey $\operatorname{GM}(1,1)$ prediction model are as follows:

2.2.1. Data Processing. For a given raw data sequence, the $\operatorname{GM}(1,1)$ model of a first-order differential equation is generated by one accumulation, and the original sequence is processed as follows:

$$
X^{(1)}=\left(x^{(1)}(1), x^{(1)}(2), \ldots x^{(1)}(n)\right)
$$

where $X^{(1)}$ is 1 -AGO sequence of $X^{(0)}$.

\subsubsection{Construction of Differential Equations and Calculation} of Parameters. Accumulate the time series of samples and construct the grey differential equation as follows:

$$
\begin{aligned}
\frac{\mathrm{d} x^{(1)}}{\mathrm{d} t}+a x^{(1)} & =b, \\
Z^{(1)} & =\left(z^{(1)}(1), z^{(1)}(2), \ldots z^{(1)}(n)\right),
\end{aligned}
$$

where $a$ is the development coefficient of the model, reflecting the trend of $x^{(1)}$ and $x^{(0)}$, and $\mathrm{u}$ is the grey action of the model, reflecting the change relationship between data.

If $\widehat{\alpha}=(a, b)^{T}$ is a parameter column, then 


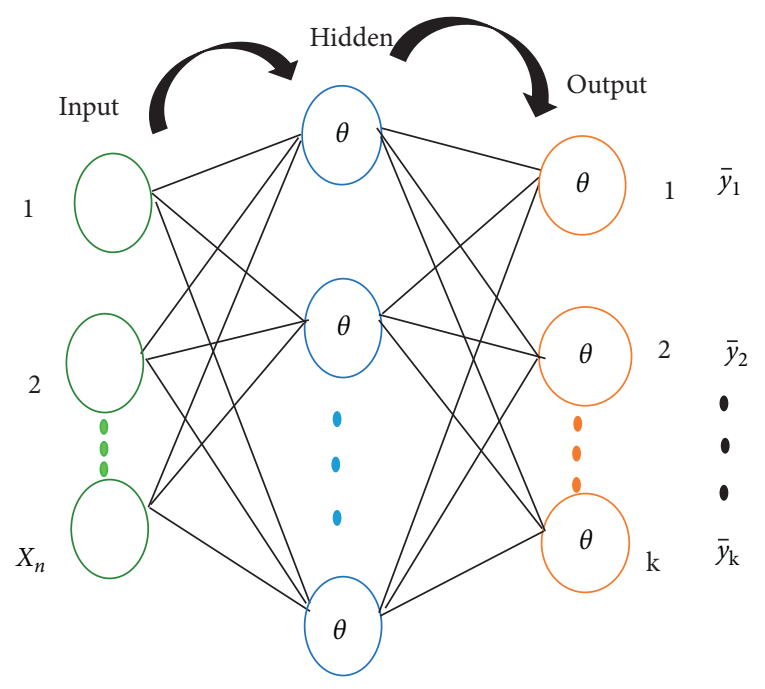

Figure 1: Neural network diagram.

$$
\begin{aligned}
Y & =\left[\begin{array}{c}
x^{(0)}(2) \\
x^{(0)}(3) \\
\cdots \\
x^{(0)}(\mathrm{n})
\end{array}\right] B \\
& =\left[\begin{array}{ccc}
-z^{(1)}(2) & 1 \\
-z^{(1)}(3) & 1 \\
\cdots & \cdots & \\
-z^{(1)}(n) & 1
\end{array}\right] .
\end{aligned}
$$

2.2.3. Model Construction and Solution. The least-squares estimation parameter sequence of a grey differential equation $x^{(0)}(k)+a z^{(1)}(k)=b$ satisfies

$$
\widehat{\alpha}=\left(B^{T} B\right)^{-1} B^{T} Y \text {. }
$$

The time response sequence of $\operatorname{GM}(1,1)$ grey differential equation is $\hat{x}^{(1)}(k+1)$ for $x^{(1)}(0)=x^{(0)}(1)$; then,

$$
\hat{x}^{(1)}(k+1)=\left[x^{(0)}-\frac{b}{a}\right] e^{-a k}+\frac{b}{a} \text {. }
$$

The predicted data $\hat{x}^{(1)}(k+1)$ is reduced to the predicted value as follows:

$$
\widehat{x}^{(0)}(k+1)=\hat{x}^{(1)}(k+1)-\widehat{x}^{(1)}(k) .
$$

2.3. Grey Markov Model. The basic Markov model is

$$
x(k+1)=x(k) \cdot R(1),
$$

where $x(k)$ represents the state of time $t=k, R(1)$ represents a one-step transition probability matrix, and $x(k+1)$ represents the state of time $t=k+1$. The grey Markov chain model is based on the prediction results of the grey prediction model to predict the correct stationary process of the overall trend, and then the Markov model is used to optimize and correct the prediction results. [27] The flow chart of the grey Markov model is shown in Figure 2.

The details of the Grey Markov model are introduced briefly as follows:

Step1: the result state division of $\operatorname{GM}(1,1)$ model prediction

The fitting data obtained by the model are compared with the actual sample data, and their relative values are used as the correction value $\lambda$ $(k)$ for interval division. Each interval represents a state [28].

$$
\lambda(k)=\frac{\widehat{x}^{(0)}(k)}{x^{(0)}(k)},
$$

$$
E i \in\left[Q_{i 1}, Q_{i 2}\right], \quad i=1,2, \ldots, k \text {, }
$$

where $Q_{i 1}$ represents the lower limit of the relative value in the state interval and $Q_{i 2}$ represents the upper limit of the relative value in the state interval.

Step2 : construction of the state probability matrix

According to the interval state of the prediction results, the one-step transition probability is determined, and the state matrix of the research system is constructed [27-30]. The state transition probability matrix of the above model is as follows:

$$
p(k)=\left[\begin{array}{cccc}
p 11(k) & p 21(k) & \cdots & p 1 n(k) \\
p 21(k) & p 22(k) & \cdots & p 2 n(k) \\
\cdots & \cdots & \cdots & \cdots \\
p n 1(k) & p n 2(k) & \cdots & p n n(k)
\end{array}\right] .
$$

Step3 : model correction

The residuals between the measured value and the simulation value are calculated, and the average residuals of all states in each state interval are taken to correct the predicted value of the grey Markov model.

\section{Model Establishment and Solution}

3.1. Prediction of Mine Dust Concentration Based on the ARIMA Model. Taking the dust concentration of 5424 working face in a mine as the original data source [12], the cubic spline interpolation method was used to interpolate the data collected between 9:31 and 10:17, and the model was established, so that the data were sufficient to support the analysis. Since the original sequence is a nonstationary sequence [29], the second-order difference method is selected to smooth the original data, and the time sequence diagram is drawn. It is found that the difference data fluctuate up and down at a certain value, so the sequence is considered to be stable. Take 2 in ARIMA model $d$. The sequence diagram after the difference is shown in Figure 3. 


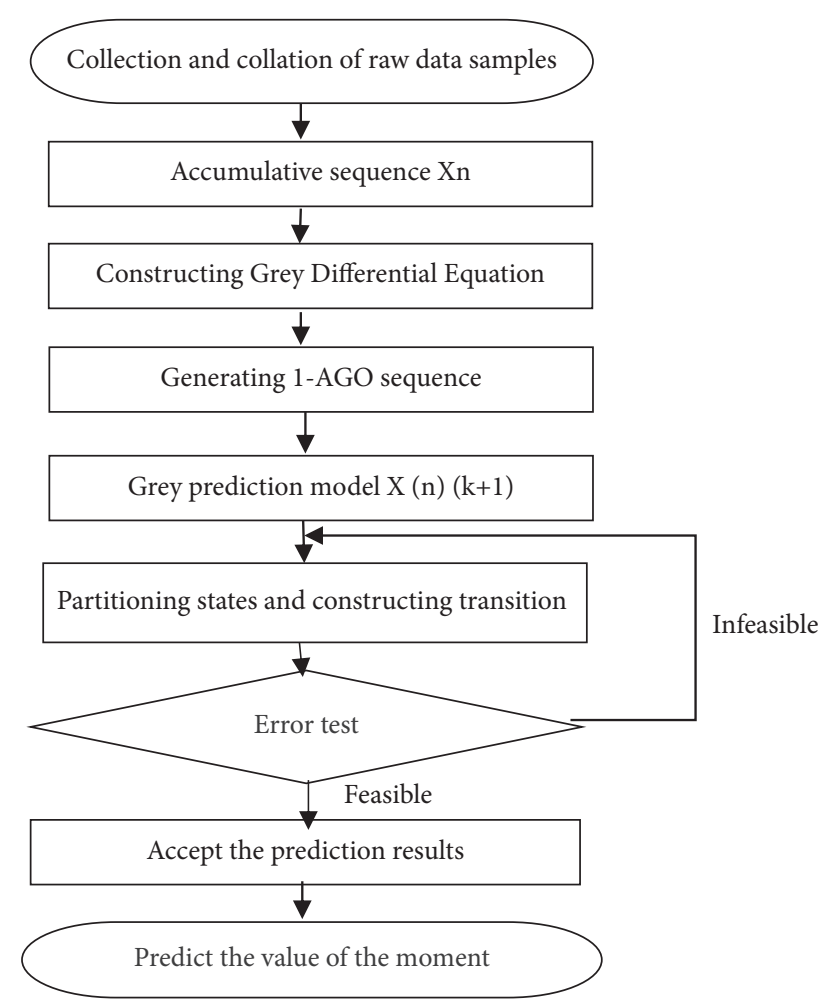

Figure 2: Grey Markov model process.

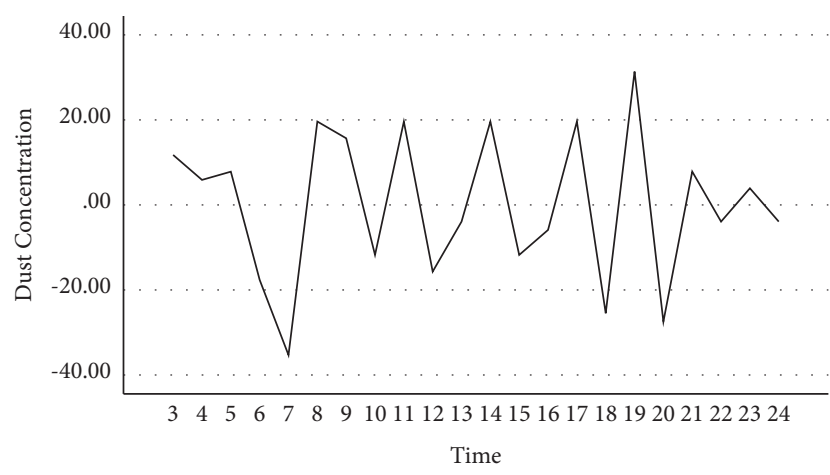

FIgURE 3: Sequence diagram after difference.

Establish the original time series model as follows:

$$
\left(1-\sum_{i=1}^{p} \alpha_{i} L^{i}\right)(1-L)^{d} y_{t}=\alpha_{0}+\left(1+\sum_{i=1}^{q} \beta_{i} L^{i}\right) \varepsilon_{t} .
$$

$L$ is a delay operator of $y$, satisfying

$$
L^{i} y_{t}=y_{t-i} .
$$

Calculated values of $p, d, q$ are 1,2 , and 1 , respectively. Formula (10) can be reduced to

$$
y_{t}=\frac{\alpha_{0}+\left(1+\beta_{1} L^{1}\right) \varepsilon_{t}}{(1-L)^{2}\left(1-\alpha_{2} L^{2}\right)} .
$$

The fitting degree of the ARIMA model is given by SPSS. The results are shown in Table 1.
TABle 1: ARIMA model-fitting degree.

\begin{tabular}{lcccc}
\hline Fitting statistics & Average & Standard error & Min & Max \\
\hline Smooth R & 0.444 &. & 0.444 & 0.444 \\
R & -0.238 &. & -0.238 & -0.238 \\
RMSE & 14.326 &. & 14.326 & 14.326 \\
MAPE & 34.244 &. & 34.244 & 34.244 \\
MAE & 9.792 &. & 9.792 & 9.792 \\
Normalizing BIC & 5.886 &. & 5.886 & 5.886 \\
\hline
\end{tabular}

Mine dust concentration is predicted by $\operatorname{ARIMA}(1,2,1)$ model. The forecast results of ARIMA are shown in Figure 4.

3.2. Prediction of Dust Concentration in Mines Based on the Neural Network Model. From the change trend of dust concentration reflected in Figure 3, the time factor occupies a great weight in the influencing factors of dust concentration change. The relationship between the concentration change trend and the time variable is not a simple linear relationship, so the neural network model is used to predict this variable.

3.2.1. Determination of Hidden Layers. The hidden layer is responsible for automatically learning the input feature information, and the multilayered hidden layer is a complex network to solve complex problems. With the increase of network layers, the accuracy and speed of calculation will also be improved $[30,31]$. However, the complexity of the model also increases, and the risk of overfitting increases. In order to improve the fitting degree of the model prediction results, considering that the actual data sample size is small and it is not easy to overfit, this paper selects a ten-layer network to establish the model.

3.2.2. Selection of Activation Functions. The essence of continuous learning of neural networks is to optimize its model parameters in receiving the feedback of continuous training errors. Among them, the error of the current layer is closely related to the selection of the activation function $[32,33]$. Since the derivative values of the sigmoid function and tanh function are not greater than 1, the gradient will inevitably disappear in the iterative process, leading to the premature termination of training. The ReLU function can well avoid this phenomenon, so the ReLU function is selected as the activation function of the model in this paper.

3.2.3. Selection of Training Algorithm. In the process of neural network training, Levenberg-Marquardt, Bayesian regularization, and quantitative conjugate gradient training algorithms are generally used for training. The sample data used in this paper is less, not easy to appear overfitting phenomenon, and the training time is short, in order to improve the fitting degree; this paper uses the training method of regularization. The results of neural network model prediction are shown in Table 2.

The weights and thresholds of neural network initialization are random, resulting in different results each time, so experiments are repeated. The prediction results were 


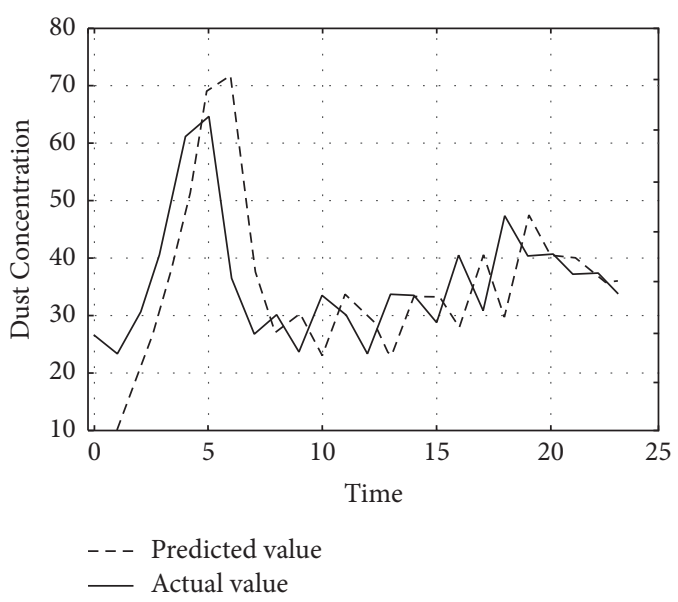

FIgURE 4: Forecasting results of the ARIMA model.

TABLE 2: Prediction value of BP neural network.

\begin{tabular}{lcccc}
\hline Number & Concentration & Pre1 & Pre2 & Pre3 \\
\hline 1 & 19.6100 & 16.5789 & 19.6552 & 22.7850 \\
2 & 15.5775 & 16.5943 & 15.4312 & 23.1299 \\
3 & 15.6900 & 16.7072 & 15.6100 & 23.7446 \\
4 & 18.7425 & 17.5014 & 18.4569 & 24.7542 \\
5 & 23.5300 & 22.3078 & 23.3166 & 26.2917 \\
6 & 29.3186 & 37.1274 & 30.0419 & 28.7765 \\
$\ldots$ & $\ldots$ & $\ldots$ & $\ldots$ & $\ldots$ \\
42 & 33.7009 & 33.2702 & 48.4017 & 44.6074 \\
43 & 31.3700 & 31.9421 & 58.3134 & 56.7107 \\
44 & 30.9197 & 31.8737 & 65.1613 & 61.7435 \\
45 & 31.3700 & 31.8647 & 63.2409 & 59.2420 \\
46 & 30.8403 & 30.8150 & 48.7600 & 49.8708 \\
47 & 27.4500 & 27.3146 & 30.7901 & 35.2170 \\
\hline
\end{tabular}

obtained by BP neural network, and the optimal model was selected in the three experiments.

BP neural network model prediction results of the first group of data relative error is 0.1418 ; mean square relative error is 0.0353 ; the second group of prediction results relative error is 0.0874 ; mean square relative error is 0.0269 ; the third group of prediction results relative error is 0.1566 ; and mean square relative error is 0.0410 . It can be seen that the second group has the best prediction results.

\subsection{Prediction of Dust Concentration Based on the Grey Markov Model}

3.3.1. Dust Concentration Model Based on Metabolic $G M(1,1)$. Taking the dust concentration of 5424 working face in a mine as the original data source [12], the cubic spline interpolation method was used to interpolate the data collected between 9:31 and 10:17, and the metabolic model was established. According to the comparison between the predicted results of the model and the actual values, the model prediction results were finally obtained. Build the original sequence according to the mine dust concentration measured every two minutes and accumulate it once to get $X^{(1)}$. Matrix B is calculated from formula (3):

$$
B=\left[\begin{array}{cc}
-9.8050 & 1 \\
-17.5938 & 1 \\
\vdots & \vdots \\
-701.833 & 1
\end{array}\right] Y=\left[\begin{array}{c}
15.58 \\
15.69 \\
\vdots \\
27.45
\end{array}\right]
$$

Calculate available by formulas (3)-(5) using MATLAB $a=0.0013172$ and grey action $b=28.5269$. Five groups of development coefficient and grey action will appear in the fourth phase of backward prediction.

Bring the $a, b$ parameters into formula (6):

$$
\begin{aligned}
\widehat{x}^{(1)}(k+1) & =\left[x^{(0)}-\frac{b}{a}\right] e^{-a k}+\frac{b}{a} \\
& =-21637.6175 e^{-0.0013172 k}+21657.2275 .
\end{aligned}
$$

The simulation value of mine dust concentration generated by the GM $(1,1)$ model is simulated by MATLAB, and the results are shown in Table 3 .

From the GM $(1,1)$ model, it can be predicted that the dust concentrations in the following four periods are $30.3562 \mathrm{~g} / \mathrm{cm}^{3}$, $30.3962 \mathrm{~g} / \mathrm{cm}^{3}, 30.4363 \mathrm{~g} / \mathrm{cm}^{3}$, and $30.4764 \mathrm{~g} / \mathrm{cm}^{3}$, respectively. The total relative error of the grey prediction model is 0.2849 , and the total mean square error is 0.1431 .

3.3.2. Numerical Simulation of the Grey Markov Model. Division of Results for $\operatorname{GM}(1,1)$ Model Prediction. Based on the mine dust concentration index from 9:31 to 10:17, the relative value between the actual concentration and the predicted value of the grey prediction model is calculated. The upper and lower limits of the relative value are divided into intervals, and the interval distance is equalized. Among them, the distribution area of relative values is relatively concentrated in [0.52 and 2.28]. The above data are divided into four states by selecting $0.52,0.96,1.40,1.84$, and 2.28 as the critical values, as shown in Table 4 .

Construction of State Probability Matrix. The state divided by Table 2 can be represented as follows:

$$
\begin{aligned}
& M 1=23, \\
& M 2=18, \\
& M 3=3, \\
& M 4=3 .
\end{aligned}
$$

In the calculation of the transition probability matrix $P$, it is impossible to determine the next state of the last state turning, so it not included in the calculation. One-step transition frequency matrix and one-step transition probability matrix are as follows: 
TAble 3: Prediction of mine dust concentration by the $\operatorname{GM}(1,1)$ model.

\begin{tabular}{lccccc}
\hline Number & Concentration & $\begin{array}{c}\text { GM }(1, \\
1)\end{array}$ & $\begin{array}{c}\text { Relative } \\
\text { error }\end{array}$ & $\begin{array}{c}\text { Relative } \\
\text { value }\end{array}$ & Stata \\
\hline 1 & 19.61 & 19.61 & 0 & 1 & E2 \\
2 & 15.5775 & 28.5716 & 0.834158 & 0.545209 & E1 \\
3 & 15.69 & 28.6092 & 0.823403 & 0.548425 & E1 \\
4 & 18.7425 & 28.647 & 0.528451 & 0.654257 & E1 \\
5 & 23.53 & 28.6847 & 0.219069 & 0.820298 & E1 \\
6 & 29.3186 & 28.7225 & 0.0203 & 1.0208 & E2 \\
7 & 37.2600 & 28.7604 & 0.2281 & 1.2955 & E2 \\
8 & 48.0679 & 28.7983 & 0.4009 & 1.6691 & E3 \\
9 & 58.8200 & 28.8362 & 0.5098 & 2.0398 & E4 \\
10 & 65.4248 & 28.8742 & 0.5587 & 2.2659 & E4 \\
$\ldots$ & $\ldots$ & $\ldots$ & $\ldots$ & $\ldots$ & $\ldots$ \\
37 & 43.1400 & 29.9196 & 0.0825 & 1.0899 & E2 \\
38 & 41.5041 & 29.9590 & 0.3065 & 1.4419 & E3 \\
39 & 35.2900 & 29.9985 & 0.2782 & 1.3854 & E2 \\
40 & 34.2565 & 30.0380 & 0.1499 & 1.1764 & E2 \\
41 & 35.2900 & 30.0776 & 0.1231 & 1.1404 & E2 \\
42 & 33.7009 & 30.1173 & 0.106335 & 1.118988 & E2 \\
43 & 31.37 & 30.157 & 0.038668 & 1.040223 & E2 \\
44 & 30.9197 & 30.1967 & 0.023383 & 1.023943 & E2 \\
45 & 31.37 & 30.2365 & 0.036133 & 1.037488 & E2 \\
46 & 30.8403 & 30.2764 & 0.018285 & 1.018625 & E2 \\
47 & 27.45 & 30.3163 & 0.104419 & 0.905454 & E1 \\
\hline & & & & &
\end{tabular}

$$
\begin{aligned}
\left(f_{i j}\right)_{4 \times 4} & =\left[\begin{array}{cccc}
18 & 4 & 0 & 0 \\
5 & 11 & 2 & 0 \\
0 & 2 & 0 & 1 \\
0 & 0 & 1 & 2
\end{array}\right], \\
P(1) & =\left[\begin{array}{cccc}
\frac{9}{11} & \frac{2}{11} & 0 & 0 \\
\frac{5}{18} & \frac{11}{18} & \frac{1}{9} & 0 \\
0 & \frac{2}{3} & 0 & \frac{1}{3} \\
0 & 0 & \frac{1}{3} & \frac{2}{3}
\end{array}\right] .
\end{aligned}
$$

$P(1), P(2), P(3)$, and $P(4)$ matrices are obtained by using a four-step transfer probability matrix to calculate 10 : 19 dust concentration data. Select the nearest four time points: 10:17, 10:16, 10:15, and 10:14; according to the distance from the time point in turn to take the number of transfer steps in the transfer matrix corresponding to the number of transfer steps in the initial state of the corresponding row vector to form a new probability matrix and
TABLE 4: State partition table.

\begin{tabular}{lcc}
\hline State & Interval range & Number \\
\hline E1 & {$[0.52,0.96]$} & 23 \\
E2 & {$[0.96,1.4]$} & 18 \\
E3 & {$[1.4,1.84]$} & 3 \\
E4 & {$[1.84,2.28]$} & 3 \\
\hline
\end{tabular}

the column vector summation of the probability matrix to determine the future steering state of the system, the largest sum is the state of $\mathrm{E} 2$. The results of mine dust concentration prediction at 10:19 are shown in Table 5 .

The probability of state E2 is the highest in the total row in Table 3, so it can be predicted that dust concentration at 10:19 is in state E2. The predicted value of the grey Markov chain is as follows:

$$
\widehat{x}(t)=[(0.96+1.4) \times 30.3562] \div 2=35.820316 .
$$

The prediction value and relative error of dust concentration under the ARIMA model and grey Markov chain model are listed in Table 6.

The average interval data are used to replace the Markov prediction interval as its predicted value. At the same time, ARIMA $(1,2,1)$ model was used to predict the dust concentration of mine, and the prediction results were compared with those of the grey Markov model. The results show that the overall relative error of the ARIMA model is 0.1865 , the total mean square relative error is 0.0348 , the overall relative error of the grey Markov model is 0.1262 , and the total mean square relative error is 0.0259 . The comparison results of prediction accuracy of the three models show that the prediction effect of the GM $(1,1)$ model is the most unsatisfactory, the prediction effect of the ARIMA model is in the middle, and the prediction effect of the grey Markov model is the best.

It can be seen from Figure 5 that the predicted values of dust concentration during the period from 9:31 to 10:17 obtained by the four models are compared with the actual values during this period. It can be obviously seen that the values predicted by the grey Markov model are closer to the actual values of comprehensive evaluation. Therefore, it is concluded that the grey Markov model can well fit the development trend and volatility characteristics of the sequence.

\section{Residual Test}

4.1. Evaluation Indexes of Forecasting Performance. To compare the forecast obtained using the proposed model with those obtained using other models, the mean absolute percentage error (MAPE), the root mean squared error (RMSE), and the mean absolute error (MAE) are used to evaluate forecasting accuracy. 
TABle 5: 10:19 Prediction of mine dust concentration.

\begin{tabular}{lcccccc}
\hline Time & Initial state & Transfer steps & E1 & E2 & E3 & E4 \\
\hline $10: 17$ & E2 & 1 & 0.2778 & 0.6111 & 0.1111 & 0 \\
$10: 16$ & E2 & 2 & 0.3970 & 0.4980 & 0.0679 & 0.0370 \\
$10: 15$ & E2 & 3 & 0.4631 & 0.4218 & 0.0677 & 0.0473 \\
$10: 14$ & E2 & 4 & 0.4961 & 0.3871 & 0.0626 & 0.0541 \\
& Total & & 1.634 & 1.918 & 0.3093 & 0.1384 \\
\hline
\end{tabular}

TABle 6: Comparison between ARIMA model and grey Markov model.

\begin{tabular}{|c|c|c|c|c|c|c|c|}
\hline Number & Concentration & ARIMA & Relative error & Mean square relative error & Grey Markov & Relative error & Mean square relative error \\
\hline 1 & 19.6100 & & & & 23.1398 & 0.1800 & 0.0324 \\
\hline 2 & 15.5775 & & & & 21.1430 & 0.3573 & 0.1276 \\
\hline 3 & 15.6900 & 15.5900 & 0.0064 & 0.0000 & 21.1708 & 0.3493 & 0.1220 \\
\hline 4 & 18.7425 & 13.5700 & 0.2760 & 0.0762 & 21.1988 & 0.1311 & 0.0172 \\
\hline 5 & 23.5300 & 23.7200 & 0.0081 & 0.0001 & 21.2267 & 0.0979 & 0.0096 \\
\hline 6 & 29.3186 & 24.7500 & 0.1558 & 0.0243 & 33.8926 & 0.1560 & 0.0243 \\
\hline 7 & 37.2600 & 34.8100 & 0.0658 & 0.0043 & 33.9373 & 0.0892 & 0.0080 \\
\hline 8 & 48.0679 & 39.8800 & 0.1703 & 0.0290 & 46.6532 & 0.0294 & 0.0009 \\
\hline 9 & 58.8200 & 48.0300 & 0.1834 & 0.0337 & 59.4026 & 0.0099 & 0.0001 \\
\hline 10 & 65.4248 & 61.6400 & 0.0578 & 0.0033 & 59.4809 & 0.0909 & 0.0083 \\
\hline 11 & 62.7500 & 53.3300 & 0.1501 & 0.0225 & 59.5593 & 0.0508 & 0.0026 \\
\hline 12 & 48.3254 & 64.3600 & 0.3318 & 0.1101 & 46.8996 & 0.0295 & 0.0009 \\
\hline 13 & 31.3700 & 49.3200 & 0.5722 & 0.3274 & 34.2065 & 0.0904 & 0.0082 \\
\hline 14 & 21.6723 & 34.4300 & 0.5887 & 0.3465 & 21.4798 & 0.0089 & 0.0001 \\
\hline 15 & 19.6100 & 34.4800 & 0.7583 & 0.5750 & 21.5081 & 0.0968 & 0.0094 \\
\hline 16 & 22.3377 & 21.9800 & 0.0160 & 0.0003 & 21.5364 & 0.0359 & 0.0013 \\
\hline 17 & 23.5300 & 21.5100 & 0.0858 & 0.0074 & 21.5649 & 0.0835 & 0.0070 \\
\hline 18 & 18.8867 & 22.9300 & 0.2141 & 0.0458 & 21.5933 & 0.1433 & 0.0205 \\
\hline 19 & 15.6900 & 21.3900 & 0.3633 & 0.1320 & 21.6218 & 0.3781 & 0.1429 \\
\hline 20 & 20.7556 & 15.8700 & 0.2354 & 0.0554 & 21.6503 & 0.0431 & 0.0019 \\
\hline 21 & 27.4500 & 20.6000 & 0.2495 & 0.0623 & 21.6787 & 0.2102 & 0.0442 \\
\hline 22 & 28.0011 & 26.1800 & 0.0650 & 0.0042 & 21.7074 & 0.2248 & 0.0505 \\
\hline 23 & 23.5300 & 21.2400 & 0.0973 & 0.0095 & 21.7359 & 0.0762 & 0.0058 \\
\hline 24 & 17.7300 & 22.3700 & 0.2617 & 0.0685 & 21.7646 & 0.2276 & 0.0518 \\
\hline 25 & 15.6900 & 22.9500 & 0.4627 & 0.2141 & 21.7933 & 0.3890 & 0.1513 \\
\hline 26 & 20.6990 & 16.2600 & 0.2145 & 0.0460 & 21.8220 & 0.0543 & 0.0029 \\
\hline 27 & 27.4500 & 21.3700 & 0.2215 & 0.0491 & 21.8508 & 0.2040 & 0.0416 \\
\hline 28 & 29.8742 & 26.2500 & 0.1213 & 0.0147 & 34.8891 & 0.1679 & 0.0282 \\
\hline 29 & 27.4500 & 23.2800 & 0.1519 & 0.0231 & 21.9084 & 0.2019 & 0.0408 \\
\hline 30 & 22.2993 & 26.1400 & 0.1722 & 0.0297 & 21.9373 & 0.0162 & 0.0003 \\
\hline 31 & 21.5700 & 27.4300 & 0.2717 & 0.0738 & 21.9662 & 0.0184 & 0.0003 \\
\hline 32 & 29.7035 & 22.1500 & 0.2543 & 0.0647 & 35.0734 & 0.1808 & 0.0327 \\
\hline 33 & 35.2900 & 25.4500 & 0.2788 & 0.0777 & 35.1196 & 0.0048 & 0.0000 \\
\hline 34 & 28.7318 & 33.5300 & 0.1670 & 0.0279 & 35.1659 & 0.2239 & 0.0501 \\
\hline 35 & 23.5300 & 31.4700 & 0.3374 & 0.1139 & 22.0823 & 0.0615 & 0.0038 \\
\hline 36 & 32.5657 & 24.4400 & 0.2495 & 0.0623 & 35.2586 & 0.0827 & 0.0068 \\
\hline 37 & 43.1400 & 32.0500 & 0.2571 & 0.0661 & 48.4698 & 0.1235 & 0.0153 \\
\hline 38 & 41.5041 & 41.5900 & 0.0021 & 0.0000 & 35.3516 & 0.1482 & 0.0220 \\
\hline 39 & 35.2900 & 35.9400 & 0.0184 & 0.0003 & 35.3982 & 0.0031 & 0.0000 \\
\hline 40 & 34.2565 & 35.0500 & 0.0232 & 0.0005 & 35.4448 & 0.0347 & 0.0012 \\
\hline 41 & 35.2900 & 36.0800 & 0.0224 & 0.0005 & 35.4916 & 0.0057 & 0.0000 \\
\hline 42 & 33.7009 & 35.4800 & 0.0528 & 0.0028 & 35.5384 & 0.0545 & 0.0030 \\
\hline 43 & 31.3700 & 33.6800 & 0.0736 & 0.0054 & 35.5853 & 0.1344 & 0.0181 \\
\hline 44 & 30.9197 & 31.7000 & 0.0252 & 0.0006 & 35.6321 & 0.1524 & 0.0232 \\
\hline 45 & 31.3700 & 31.3500 & 0.0006 & 0.0000 & 35.6791 & 0.1374 & 0.0189 \\
\hline 46 & 30.8403 & 31.3900 & 0.0178 & 0.0003 & 35.7262 & 0.1584 & 0.0251 \\
\hline 47 & 27.4500 & 29.8000 & 0.0856 & 0.0073 & 22.4341 & 0.1827 & 0.0334 \\
\hline
\end{tabular}




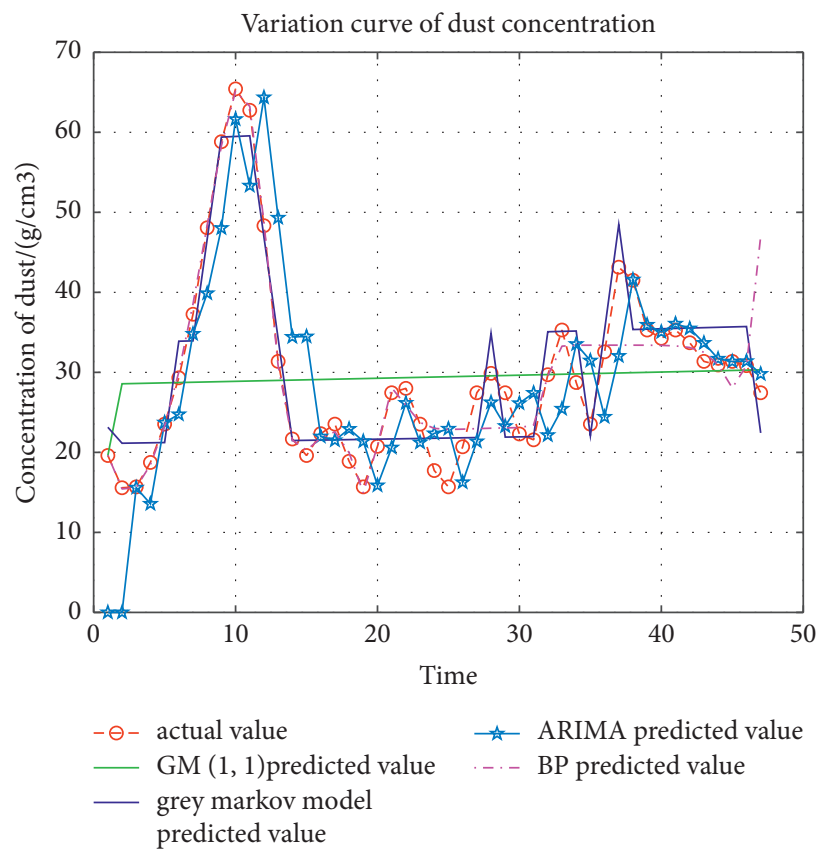

FIGURE 5: Comparison of measured and predicted dust concentration.

TABLE 7: Comparison of prediction accuracy of the above models.

\begin{tabular}{lccc}
\hline Model & MAE & MAPE & RMSE \\
\hline GM $(1,1)$ & 8.12 & 0.28 & 11.58 \\
Grey Markov & 3.26 & 0.13 & 3.87 \\
ARIMA & 5.19 & 0.19 & 6.82 \\
Neural network & 2.35 & 0.09 & 4.31 \\
\hline
\end{tabular}

TABLE 8: Classification table of prediction accuracy.

\begin{tabular}{lcc}
\hline Probability of small error $(P)$ & Mean variance ratio $(C)$ & Prediction accuracy \\
\hline$>0.95$ & $<0.35$ & Excellent \\
$>0.80$ & $<0.50$ & Qualification \\
$>0.70$ & $<0.65$ & Reluctance \\
$\leq 0.70$ & $\geq 0.65$ & Nonconformity \\
\hline
\end{tabular}

$$
\begin{aligned}
\text { MAPE } & =\frac{1}{N} \sum_{i=1}^{N}\left|\frac{y_{i}-f_{i}}{y_{i}}\right| x 100 \%, \\
\text { RMSE } & =\sqrt{\frac{\sum_{i=1}^{N}\left(y_{i}-f_{i}\right)^{2}}{N}}, \\
\text { MAE } & =\frac{\sum_{i=1}^{N}\left(y_{i}-f_{i}\right)^{2}}{N},
\end{aligned}
$$

where $N$ is the total number of forecasts, $y_{i}$ is the actual value at forecasting point $i$, and $f_{i}$ is the forecasted value at forecasting point $i$. A comparison of prediction accuracy of the above models is shown in Table 7.

The comparison of the prediction accuracy of the above four models shows that the grey Markov model and the neural network model have better prediction accuracy, and their prediction accuracy is much higher than that of the other two models.
4.2. Residual Verification. The posterior error ratio $C$ and small error probability $P$ test were used to test the pros and cons of the prediction model and the accuracy level. The accuracy of the results is calculated by the above two prediction models. The accuracy classification table is shown in Table 8:

The grey Markov model considers the dynamic transfer influence components; the dynamic transfer influence components reflect the influence degree of random factors and volatility factors, which has the advantage of predicting large random volatility problems. The variance ratio of $\operatorname{GM}(1,1)$ model is $C=0.9928$, and that of small probability error is $P=0.5532$. The variance ratio of Grey Markov model is $C=0.3256$, and that of small probability error is $P=1$. The accuracy of four prediction models is compared as shown in Table 9.

It can be seen from Table 9 that the prediction accuracy of the grey Markov model is higher than that of $\operatorname{GM}(1,1)$ model. The prediction of mine dust concentration by grey 
TABLE 9: Comparison of prediction accuracy between four prediction models.

\begin{tabular}{lccc}
\hline Forecasting model & $\begin{array}{c}\text { Probability of small error } \\
(P)\end{array}$ & $\begin{array}{c}\text { Mean variance ratio } \\
(C)\end{array}$ & Prediction accuracy \\
\hline ARIMA & 0.7234 & 0.5879 & Reluctance \\
Neural network & 0.9149 & 0.3699 & Qualification \\
GM $(1,1)$ & 0.5532 & 0.9928 & Nonconformity \\
Grey Markov model & 1 & 0.3256 & Excellent \\
\hline
\end{tabular}

Markov model is more reliable and accurate and can accurately describe the state of mine dust concentration.

\section{Conclusion}

The exploration of dust concentration changing with time in mines has been one of the research hotspots in the field of mine safety. Dust is extremely harmful to human health, industrial production, and living environment. It is of great significance to accurately predict the dust concentration by building a model. The following conclusions can be drawn based on the study of the dust concentration in mines:

(1) The Markov chain theory is used to optimize the GM $(1,1)$ model. According to the range of relative error generated by GM $(1,1)$ model prediction, the state interval is reasonably divided, and the corresponding state transition probability matrix is determined. The GM $(1,1)$ model based on Markov optimization is established.

(2) The model combines the advantages of the grey prediction model and the Markov model to improve the fitting accuracy of prediction objects with large random fluctuation. The experimental results show that the grey Markov model has better prediction accuracy than grey prediction, ARIMA, and neural network models.

(3) In the analysis of the prediction results of mine dust concentration, it can be concluded that the grey Markov model is suitable for short-term prediction with less sample data. The experimental results show that this model is more accurate than the traditional model. It has great application space in mine dust concentration prediction.

\section{Data Availability}

The dust concentration of 5424 working face in a mine as the original data source used to support the findings of this study is included within the article.

\section{Conflicts of Interest}

The authors declare that they have no conflicts of interest.

\section{Acknowledgments}

This work was supported by the National Natural Science Foundation of China (grant no. 51974128); Hebei Province Doctoral Student Innovation Funding Project (CXZZBS2021102); and Basic Scientific Research Business
Expenses of Provincial Universities of the North China University of Technology in 2021 (JQN2021011).

\section{References}

[1] W. Lianjiang, J. Kang, X. Song, F. Zhou, and K. Chen, "Evaluation index and classification method of mine ventilation difficulty," Journal of China University of Mining and Technology, vol. 50, no. 4, pp. 649-657, 2021.

[2] G. Kan, "Discussion on mine dust hazards and prevention," Shandong Coal Science and Technology, vol. 195, no. 11, pp. $68-70+73,2016$

[3] B. Wang, "The prediction of dust concentration in fully mechanized mining face of coal roadway based on neural network," Mining Research and Development, vol. 37, no. 6, pp. 22-25, 2017.

[4] G. Zhao and F. Ma, "Particle swarm optimization algorithm optimizes the dust concentration prediction of BP neural network," Measurement and control technology, vol. 37, no. 6, pp. 20-23, 2018.

[5] Ke Li, Study on Quantitative Simulation and Health hazard Assessment of Dust in Construction Stage of Building Engineering, East China Jiaotong University, Nanchang, China, 2020.

[6] Y. Wang, X. Li, F. Chai, J. Yuan, S. Yin, and J. Wu, "The grey generalized regression neural network combination model was used to predict the number of pneumoconiosis patients in China," Environmental and occupational medicine, vol. 33, no. 10, pp. 984-987 + 999, 2016.

[7] Z. Li, "Grey prediction of mine safety and dustproof index," Industrial Safety and Dustproof, no. 12, pp. 10-11, 1994.

[8] X. Zhao, Comprehensive Evaluation of Petrochemical Equipment Operation Status Based on Weighting Method and Grey Markov Model, Taiyuan University of Technology, Taiyuan, China, 2019.

[9] H. Wang, F. You, and B. Zhang, "Optimization simulation and application of shape PID controller based on grey prediction model," Journal of Ordnance Equipment Engineering, vol. 42, no. 10, pp. 211-217, 201.

[10] D. Yuan, X. Liu, and X. Zhang, "Research on domestic water consumption prediction based on improved grey model GM $(1,1)$," Shaanxi Water Resources, vol. 234, no. 7, pp. 1-3 + 16, 2020.

[11] Q. Wang and X. Wang, "Grey prediction model based on data optimization in Tianjin water supply prediction application," Haihe Water Conservancy, vol. 1, pp. 48-50, 2020.

[12] Y. Wang, M. Gao, and S. Zhao, "Establishment and application of mine dust concentration prediction model," China Mining, vol. 30, no. 1, pp. 178-181, 2021.

[13] Y. Liu, M. Qian, P. Guan et al., “Application of grey Markov model in prediction of discharged patients with new coronavirus pneumonia in Hubei," China Health Statistics, vol. 38, no. 3, pp. 332-335+339, 2021. 
[14] D. Shi, Study on Occupational Health Prediction Based on Grey GM ( 1,1 ) -Markov Chain Model, Tianjin University of Technology, Tianjin, China, 2017.

[15] Q. S. Dong, W. Song, G. Zhang et al., "Occupational disease prediction research based on grey GM $(1,1)$-Markov model," Safety production science and technology in China, vol. 13, no. 4, pp. 176-180, 2017.

[16] Q. Lu, Y. Wang, L. Yang, and B. Xin, "Prediction of subway passenger flow based on equal-dimensional innovation grey Markov," Safety production science and technology in China, vol. 17, no. 1, pp. 54-60, 2021.

[17] T. Wang and H. Peng, "Research on domestic production safety accident prediction based on improved GM $(1,1)$ Markov model," Industrial Engineering, vol. 23, no. 6, pp. 60-67, 2020.

[18] Z. Weng, C. Qiu, F. Qiu, Y. Yang, R. Lu, and S. He, "Grey GM $(1,1)$ settlement prediction model based on Markov optimization and its application," Science Technology and Engineering, vol. 20, no. 29, pp. 12065-12070, 2020.

[19] J. Cai, X. Wang, and Y. Cai, "Prediction of water resources demand in Beijing based on unbiased grey-Markov chain model," Three Gorges Ecological Environment Monitoring, pp. 1-15, 2021.

[20] C. Zhang, Considering Uncertainty, the Optimal Allocation of Water Resources and the harmony of Allocation System in Yuyang District of Yulin City, Northwest Agricultural and Forestry University, Xianyang, China, 2017.

[21] D. Ming, S. Wang, L. Ming, L. Ding, X. Tian, and M. Tian, "Improved dynamic frame slot ALOHA algorithm combined with BP neural network," Small microcomputer system, vol. 42, no. 9, pp. 1920-1923, 2021.

[22] H. Zeng, Z. You, X. Huang, and Y. Pu, "Application of linear regression and BP neural network in noise monitoring," Computer System Applications, vol. 30, no. 8, pp. 317-323, 2021.

[23] H. Zhang, Y. Ma, Y. Li, R. Zhang, X. Zhang, and R. Zhang, "Prediction model of walnut rupture work based on genetic BP neural network," Journal of Agricultural Engineering, vol. 30, no. 18, pp. 78-84, 2014.

[24] J. Deng, "Grey control system," Journal of Huazhong University of Technology, no. 3, pp. 9-18, 1982.

[25] A. J. SalheinKhaled and Z. Mohamed, "Output temperature predictions of the geothermal heat pump system using an improved grey prediction model," Energies, vol. 14, no. 16, 2021.

[26] J. Jiang, R. Huang, W. Xiaojing, and D. Luo, "Construction and application of consistent fractional discrete grey model," Mathematics in Practice and Knowledge, vol. 51, no. 16, pp. 128-134, 2021.

[27] C. Shi, The Improvement and Application of Grey Markov Chain, Lanzhou University, Lanzhou, China, 2014.

[28] Y. Wang and H. L. DuoxiYao, "Mine gas emission prediction based on grey Markov prediction model," Open Journal of Geology, vol. 8, no. 10, 2018.

[29] C. Liu, Y. Liang, H. Deng, Y. Guo, D. Li, and Q. Duan, "The prediction of ammonia concentration in piggery based on ARIMA and BP neural network," China Environmental Science, vol. 39, no. 6, pp. 2320-2327, 2019.

[30] Z. Xiong and X. Wang, "Dynamic unbiased grey Markov prediction of mine relative gas emission," Journal of Safety and Environment, vol. 15, no. 3, pp. 15-18, 2015.

[31] Y. Zhang, Prediction of Dust Concentration in Haer-Wusu Open-Pit Coal Mine Based on Cyclic Neural Network, China University of Mining and Technology, Beijing, China, 2020.
[32] D. Liang, The Optimization of Selective Laser Melting Process Based on Neural Network and Genetic algorithm, Xiamen Institute of Technology, Xiamen, China, 2021.

[33] F. Wang, H. Su, and K. Jing, "Dam safety monitoring model based on ARIMA-ANN," Journal of Wuhan University (Natural Science Edition), vol. 43, no. 5, pp. 585-588, 2010. 